Indian Journal of Science and Technology

http://www.indjst.org

Vol.1 No.2 (Dec. 2007)

\title{
Analytical solution to balanced quadratic cooperative game and its application to transmission loss allocation \\ RM Saloman Danaraj ${ }^{*}$, Shankarappa F Kodad ${ }^{* *}$ and Tulsi Ram Das ${ }^{* * *}$ \\ * Aurora's Technological Research Institute, Andhra Pradesh, India \\ ${ }^{* *}$ Aurora's Engineering College, Bhongir-, ${ }^{* * *}$ JNT University-, Hyderabad, Andhra Pradesh, India \\ Email: kodadsf@rediffmail.com
}

\begin{abstract}
The coalition values of the transmission loss allocation problem exhibits a specific variation and it is possible to develop analytical solution to determine the nucleolus. So an analytical solution to determine nucleolus is achieved without use of series of linear programs. It has been applied to a six-bus system and the results are compared with other methods .It is found that his method provides fair allocation and requires less computational time.
\end{abstract}

Keywords: cooperative game, transmission loss, nucleolus, power flow.

Introduction

In the deregulated power market one of the most important issues is the allocation of transmission losses among market participants since system losses can typically represent significant portion of the total generation. The main difficulty of loss allocation is caused by the highly nonlinear and non-separable properties of the loss function.

A number of allocation schemes have been proposed in the literature (Bierman \& Fernandes, 1998; Chang \& Lu, 2002; Conejo et al., 2002; Daniel et al., 2005; Ding \& Abur, 2004; Galiana \& Phelan, 2000; Gross \& Tao, 2000; Hsieh \& Wang, 2006; Leite da Silva \& Guilherme de Carvalho Costa, 2003; Shih-Chieh, 2000; Young, 1994; Zolezzi \& Rudnick, 2002). Some approaches are based on DC power flow, while some use AC load flow for matching the calculation results and actual power flows. Some schemes are branchpower-flow based, while some focus on the

\begin{tabular}{|c|c|c|c|c|}
\hline \multicolumn{5}{|c|}{ Table I } \\
\hline $\begin{array}{c}\text { Bus } \\
\text { no }\end{array}$ & $\begin{array}{c}\text { Real } \\
\text { Power } \\
\mathrm{P}(\mathrm{MW})\end{array}$ & $\begin{array}{c}\text { Reactive } \\
\text { Power } \\
\mathrm{Q}(\mathrm{MW})\end{array}$ & $\begin{array}{c}\text { Voltage } \\
\text { Mag } \\
\text { pu }\end{array}$ & $\begin{array}{c}\text { Voltage } \\
\text { Angle } \\
\text { Degrees }\end{array}$ \\
\hline 1 & 107.8755 & 15.9562 & 1.05 & 0 \\
\hline 2 & 50 & 74.3565 & 1.05 & -3.6712 \\
\hline 3 & 60 & 89.6268 & 1.07 & -4.2733 \\
\hline 4 & -70 & -70 & 0.9894 & -4.1958 \\
\hline 5 & -70 & -70 & 0.9854 & -5.2764 \\
\hline 6 & -70 & -70 & 1.0044 & -5.9475 \\
\hline \multicolumn{3}{|c|}{ Transmission loss (MW) } & \multicolumn{3}{|c|}{7.8755} \\
\hline
\end{tabular}

branch-current based allocation techniques.

Game theory provides well-behaved solution mechanisms with economic features for assessing the interaction of different participants in competitive markets and resolving the conflicts among players (Ding \& Abur, 2004). In particular, cooperative game theory is a most convenient tool to solve cost allocation problem (Zolezzi \& Rudnick, 2002). The main solution concept of cooperative game the nucleolus is found by series of linear programs. So, shapely value (Hsieh \& Wang, 2006; Shih-Chieh, 2000) is preferred to solve cooperative game problems.

Cooperative game theory has found extensive applications power system allocation problems problems, such as transmission cost allocation (Leite da Silva \& Guilherme de Carvalho Costa, 2003) and wheeling transactions (Bierman \& Fernandes, 1998) and transmission loss allocation.

In this paper a new variant of cooperative game is derived and it has been proved that to determine the analytical solution to determine the nucleolus. This method is straight forward and does not need any linear program or any optimization routine. The transmission loss is derived as function individual current injections. Two basic formulations are presented to determine individual current injections. One basic model allocates losses only to the generators and the other allocates losses to both generators and loads. The main difference is that the former treats the load demands as equivalent constant admittances based on a real-time solved AC power flow solution and accordingly the bus admittance method impedance matrix (Ybus) is then modified, while the later formulates the load demands as equivalent current injections directly form bus impedance matrix. Each current injection is then treated as an individual player of the transmission loss allocation game. The

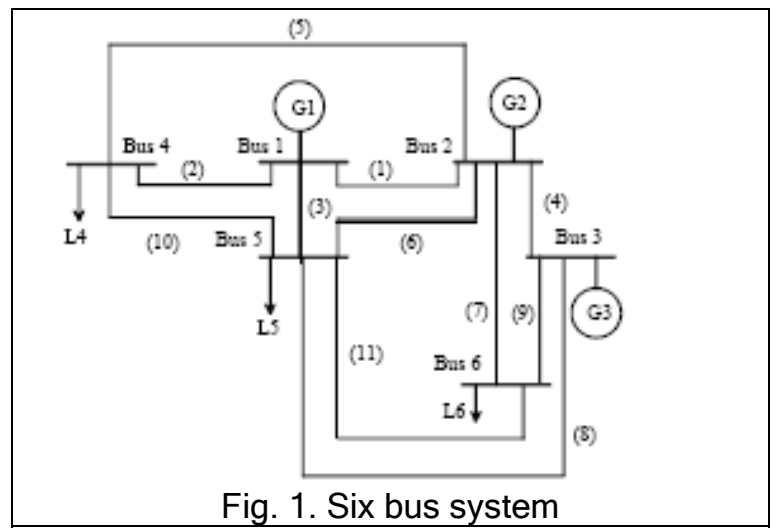


Indian Journal of Science and Technology

approaches are branch-current based, not branchpower-flow based.

The current injections (Hsieh \& Wang, 2006; Shih-Chieh, 2000) in every line of the power system obey kirchoff's law and the transmission loss is the square function of the current injections. This exhibits a specific variation and it is possible to develop analytical solution to determine the nucleolus. This is achieved without use of series of linear programs. It has been applied to a six-bus system (Fig.1) and the results are compared with other methods.

The paper is organized as follows: section II describes modeling of transmission loss problem; Section III introduces balanced quadratic cooperative game and the proof for analytical solution, and section IV about simulation and comparative study of the proposed method. This method applied to a six bus system (Tsukamoto \& lyoda, 1996) and compared with other methods in the literature.

\begin{tabular}{|c|c|c|c|c|}
\hline \multicolumn{5}{|c|}{$\begin{array}{c}\text { Table 2 } \\
\text { Transmission loss allocation by proposed method } \\
\text { (only generator buses) }\end{array}$} \\
\hline $\begin{array}{c}\text { Line } \\
\text { N0: }\end{array}$ & $\begin{array}{c}\text { G1 } \\
(\mathrm{MW})\end{array}$ & $\begin{array}{c}\text { G2 } \\
(\mathrm{MW})\end{array}$ & $\begin{array}{c}\text { G3 } \\
(\mathrm{MW})\end{array}$ & Loss \\
\hline 1 & 0.936468 & -0.03533 & 0.00380 & 0.9049 \\
\hline 2 & 0.90197 & 0.074591 & 0.11099 & 1.0875 \\
\hline 3 & 0.924853 & 0.160981 & -0.0122 & 1.0735 \\
\hline 4 & 0.005400 & -0.0414 & 0.07631 & 0.0403 \\
\hline 5 & 0.026325 & 0.810616 & 0.66816 & 1.5051 \\
\hline 6 & 0.104415 & 0.315352 & 0.07818 & 0.4979 \\
\hline 7 & 0.325988 & 0.368776 & -0.1114 & 0.5833 \\
\hline 8 & -0.02831 & 0.157133 & 0.96475 & 1.0935 \\
\hline 9 & 0.144545 & 0.139103 & 0.71973 & 1.0033 \\
\hline 10 & 0.032360 & 0.015059 & -0.0112 & 0.0362 \\
\hline 11 & 0.002090 & 0.004526 & 0.04298 & 0.0496 \\
\hline Total & 3.376109 & 1.969400 & 2.52998 & 7.8754 \\
\hline
\end{tabular}

Balanced quadratic cooperative game

Consider an ' $n$ ' person balanced linear cooperative game described by the equations (Hsieh \& Wang, 2006; Shih-Chieh, 2000). The balanced game can be described as the solution vector satisfies all the collation constraints.

$$
x(S)=v(S)
$$$$
x(N)=v(N)
$$

Where $x(S)$ is the set of possible coalitions and $x(N)$ is the grand coalition.

Let the solution vector be http://www.indjst.org

Vol.1 No.2 (Dec. 2007)

$x=\left[x_{1}^{\bullet} x_{2}^{\bullet} \ldots x_{n-1} \cdot x_{n}^{\bullet}\right]^{\mathrm{T}}$

Then second order (quadratic) cooperative game which is described as follows.

$\min \in(S)$

Subject to

$y(S) \geq(v(S))^{2}+\in(S)$

$y(N)=(v(N))^{2}$

If the solution to the game is

$y=\left[\begin{array}{llll}y_{1} & y_{2} & \cdots & \bullet y_{n-1}, y_{n}^{\bullet}\end{array}\right]^{\mathrm{T}}$

Then the relation ship between the solution vectors is

$y=v(N) \cdot x$

Proof

Multiply equations (1) by $\mathrm{v}(\mathrm{N})$

$x(S) \cdot v(N)=v(S) \cdot v(N)$

In a balanced cooperative game it is understood that

$$
\begin{gathered}
x(S) \cup x\left(S^{\prime}\right)=x(N) \\
v(S)+v\left(S^{\prime}\right)=v(N)
\end{gathered}
$$

Where $S^{\prime}$ is the conjugate of coalition $S$

$y(S)=(v(S))^{2}+v(S) \cdot v\left(S^{\prime}\right)$

$y(N)=(v(N))^{2}$

By comparing equations $(4 \& 11)$ the minimum value of the lexicographical excess vector is determined.

$e(S)=v(S) \cdot v\left(S^{\prime}\right)=e\left(S^{\prime}\right)$

Hence. it is proved and the proof can be extended to all coalition values which are real as well as complex numbers, which exhibits balancing condition. The equations $4 \& 5$ are modified for complex numbers

$$
\begin{aligned}
& y(S) \geq|v(S)|^{2}+\in(S) \\
& y(N)=|v(N)|^{2}
\end{aligned}
$$

\section{Transmission loss allocation problem}

\section{Loss allocation to generators only}

For an ' $n$ ' node power network having ' $m$ ' generator buses the transmission loss of element ij connected between nodes ' $i$ ' and ' $\mathrm{j}$ ' is derived in terms individual current contribution of each generator as

$$
{ }_{\text {loss }} P_{i j}=\left(\sum_{k=1}^{m}{ }_{i j} I_{k}\right)^{*} \cdot\left(\sum_{k=1}^{m}{ }_{i j} I_{k}\right) \cdot R_{i j}
$$


http://www.indjst.org

Vol.1 No.2 (Dec. 2007)
Indian Journal of Science and Technology

\begin{tabular}{|c|c|c|c|c|c|c|}
\hline \multicolumn{7}{|c|}{$\begin{array}{c}\text { Table 3 } \\
\text { Transmission loss allocation by proposed method } \\
\text { (Generator and load buses) }\end{array}$} \\
\hline $\begin{array}{c}\text { Line } \\
\text { N0: }\end{array}$ & G1 & G2 & G3 & L4 & L5 & L6 \\
\hline 1 & 0.8827 & -0.052 & -0.0174 & 0.02510 & 0.0024 & 0.06385 \\
\hline 2 & 0.6768 & -0.056 & -0.027 & 0.48065 & -0.019 & 0.03247 \\
\hline 3 & 0.7928 & 0.087 & -0.109 & -0.1904 & 0.3889 & 0.10466 \\
\hline 4 & 0.0072 & -0.04 & 0.0820 & 0.02430 & -0.004 & -0.032 \\
\hline 5 & -0.238 & 0.502 & 0.3442 & 1.26907 & -0.028 & -0.3443 \\
\hline 6 & 0.045 & 0.2617 & 0.0113 & -0.1490 & 0.35554 & -0.027 \\
\hline 7 & 0.182 & 0.259 & -0.261 & -0.2364 & 0.0475 & 0.59244 \\
\hline 8 & -0.088 & 0.085 & 0.8907 & 0.05907 & 0.5558 & -0.4097 \\
\hline 9 & -0.001 & -0.01 & 0.5274 & 0.00890 & -0.008 & 0.49090 \\
\hline 10 & 0.043 & 0.0177 & -0.011 & -0.0808 & 0.057 & 0.01087 \\
\hline 11 & 0.0009 & 0.0131 & 0.0546 & 0.00531 & 0.0561 & -0.0805 \\
\hline total & 2.3041 & 1.0668 & 1.4827 & 1.21561 & 1.4041 & 0.40205 \\
\hline
\end{tabular}

Since transmission loss is real the effect of shunt admittances can be ignored.

Now the current contribution of ' $k$ 'th generator to element 'ij' is given by

$$
{ }_{i j} I_{k}=\left(v_{i k}-v_{j k}\right) / Z_{i j}
$$

Where $Z_{i j}$ is the transmission line impedance of element 'ij' (pi model for transmission line is considered). It can be observed that the branch current flowing through is the algebraic sum of individual current contributions of each generator

$$
\sum_{k=1}^{n}{ }_{i j} I_{k}=I_{i j}
$$

Where ${ }^{i j} I_{k}$ is the current contribution of ' $k$ 'th generator to the element 'ij' and it can be determined from modified $Y$ bus method (Daniel et al., 2005) using converged load flow solution.

$R_{i j}$ is the resistance of line element 'ij' connected between nodes ' $\mathrm{l}$ ' and ' $\mathrm{j}$ '. The individual voltage contribution of each generator is derived in terms of current injections.

$v_{n n}=\left|\begin{array}{ll}Y_{G G} & Y_{G L} \\ Y_{L G} & Y_{L L}^{\prime}\end{array}\right|^{-1} \cdot \operatorname{diag}\left|\begin{array}{c}I_{G} \\ 0\end{array}\right|$

vnn is a square matrix of size ' $n$ ' and the columns $m+1$ to $n$ will be zero since they are load buses.

Loss allocation to generators and loads In this formulation loss allocation is made for generator as well as load buses. The individual voltage contribution of each bus is derived.

$v_{n n}=Z_{\text {bus }}$.diag $\left|I_{\text {bus }}\right|$

Now the transmission loss of 'ij'th element in terms of individual current contribution is given by

$$
{ }_{\text {loss }} P_{i j}=\left(\sum_{k=1}^{n}{ }_{i j} I_{k}\right)^{*} \cdot\left(\sum_{k=1}^{n}{ }_{i j} I_{k}\right) \cdot R_{i j}
$$

For both methods the current contributions of ' $k$ 'th bus for 'ij'th element is

$$
{ }_{i j} I_{k}=\left(v_{i k}-v_{j k}\right) / z_{i j}
$$

For each element 'ij' the coalitions present a balancing condition because of kirchoff's current law. Let 'S' be set of possible coalitions

$$
\begin{aligned}
& x(S)=I(S) \\
& x(N)=I_{i j}
\end{aligned}
$$

Let the solution vector for this balanced cooperative game is

${ }_{i j} X=\left[{ }_{i j} I_{1} \mathrm{ij}_{\mathrm{ij}} \mathrm{I}_{2} \cdots_{i j} I_{n}\right]$

Now the coalition values for the transmission loss allocation problem is derived as min e(S)

$$
\begin{aligned}
& y(S) \geq\left(I(S) \cdot I^{*}(S)\right)+e(S) \\
& y(N)=\left|I_{i j}\right|^{2} \\
& { }_{i j} y=\operatorname{real}\left(I_{i j}{ }^{*}{ }_{i j} x\right)
\end{aligned}
$$

The transmission loss contribution of ' $k$ 'th generator to 'ij'th element is determined as

$$
{ }_{i j} P_{k}={ }_{i j} y_{k} \cdot R_{i j}
$$

Now the transmission loss contribution of ' $k$ 'th generator is the summation of losses to every line element of that generator.

$$
{ }_{\text {loss }} P_{k}=\sum_{i j}{ }_{i j} P_{k}
$$

$Z_{i j}$ is the transmission line impedance of element 'ij' ( $p$ i model for transmission line is considered). 
Indian Journal of Science and Technology

http://www.indjst.org

Vol.1 No.2 (Dec. 2007)

\begin{tabular}{|c|c|c|c|c|c|c|c|c|}
\hline \multicolumn{9}{|c|}{ Table 4 Comparison of results } \\
\hline & & G1(MW) & G2(MW) & G3(MW) & L4(MW) & L5(MW) & L6(MW) & Total(MW) \\
\hline $\begin{array}{c}\text { Proposed } \\
\text { method }\end{array}$ & $\begin{array}{c}\text { Generator } \\
\text { only }\end{array}$ & 3.3761 & 1.9694 & 2.5299 & - & - & - & 7.8754 \\
\cline { 2 - 10 } & All buses & 2.3041 & 1.0668 & 1.4827 & 1.21561 & 1.4041 & 0.40205 & 7.8754 \\
\hline $\begin{array}{c}\text { Shapely } \\
\text { value[1] }\end{array}$ & $\begin{array}{c}\text { Generator } \\
\text { only }\end{array}$ & 3.37 & 1.97 & 2.53 & - & - & - & 7.8754 \\
\cline { 2 - 10 } & All buses & 2.3 & 1.07 & 1.48 & 1.22 & 1.4 & .4 & 7.875 \\
\hline
\end{tabular}

Simulation results

This method is applied to a six bus system (Wood \& Wollenberg, 1996) which has 11 line elements. The converged load flow solution is given in Table I. The loss allocation to generators buses only is given Table 2 and including load buses is given Table 3. It is compared with shapely value method (Table 4) and it is observed that the values are very close.

\section{Conclusion}

An efficient and interesting method is presented to solve a variant of cooperative game and applied to solve transmission loss allocation problem. This method offers direct solution and does not get affected by dimensionality of the problem.

Acknowledgement

The authors thank the Director and Management of Aurora Technological Research Institute, Parvathapur, Hyderabad for their support and encouragement to carry out this research.

\section{References}

1. Bierman HS and Fernandes L (1998) Game Theory with Economic Applications: AddisonWesley.

2. Chang Y-C and Lu C-N (2002) Bus-oriented transmission loss allocation. IEE. Proc.-Gener. Transm. Distrib. 149, 402-406.

3. Conejo AJ, Arroyo JM, Alguacil N, and Guijarro AL (2002) Transmission loss allocation: a comparison of different practical algorithms. IEEE Trans. Power Syst. 17, 571-576.

4. Daniel JS, Salgado RS and Irving MR (2005) Transmission loss allocation through a modified Ybus. IEE Proceedings- Generation, Transmission and Distribution. 152, 208-214.

5. Ding $Q$ and Abur A (2004) Transmission loss allocation in a multiple transaction framework. IEEE Trans. Power Syst. 19, 214-220.

6. Galiana FD and Phelan M (2000) Allocation of transmission losses to bilateral contracts in a competitive environment. IEEE Trans. Power Syst. 15,143-150.

7. Gross, G and Tao S (2000) A physical-flowbased approach to allocating transmission losses in a transaction framework. IEEE Trans. Power Syst., 15, 631-637.
8. Hsieh S-C and Wang HM (2002) Allocation of transmission losses based on cooperative game theory and current injection models. In: Proc. IEEE Int. Conf. Industrial Tech., Bangkok, Thailand, 11-14 Dec. 2002,pp. 850853.

9. Leite da Silva AM, Guilherme de Carvalho Costa J (2003) Transmission loss allocation: part I - single energy market. IEEE Trans. Power Syst. 18, 1389-1394.

10. Shih-Chieh Hsieh (2006) Fair Transmission Loss Allocation Based on Equivalent Current Injection and Shapely Value. IEEE Power Engineering Society General Meeting.

11. Tsukamoto $Y$ and lyoda I (1996) Allocation of fixed transmission cost to wheeling transactions by cooperative game theory. IEEE Trans. Power Syst. 11, 620-629.

12. Wood AJ and Wollenberg BF (199) Power Generation Operation and Control, 2nd ed., New York: Wiley, p:124.

13. Young H (1994) Cost Allocation. In: Handbook of Game Theory. vol. 2, pp. 1193-1235.

14. Zolezzi JM and Rudnick H (2002) Transmission cost allocation by cooperative games and coalition formation. IEEE Trans. Power Syst. 17, 1008-1015. 